\title{
La cinta de medir como técnica predictiva: la razón Cintura-Estatura, el síndrome metabólico y el riesgo cardiovascular.
}

\author{
Tomás Romero
}

Los intentos de interpretar el significado de la forma del cuerpo humano son tan antiguos como el hombre mismo, pero adquieren un carácter científico a partir de 1890 con las mediciones de Bertillon, Galton y Cesare Lombroso con propósitos más bien criminológicos. Posteriormente esfuerzos para establecer patrones diferenciales de inteligencia entre diferentes grupos étnicos llevaron a extremos lamentables y al descrédito de muchas de las técnicas antropométricas ${ }^{1}$. En la década de 1940 Sheldon propone su teoría de los somatotipos, según la cual las características somáticas reflejarían rasgos psicológicos y conductuales ${ }^{2}$. El interés en medir las diferentes expresiones corporales de obesidad surgió con posterioridad cuando, a través de múltiples estudios epidemiológicos, se comprobaron tanto su significado como factor importante en la morbi-mortalidad de individuos y poblaciones, al igual que la distribución abdominal del tejido adiposo, como un marcador sensible de riesgo cardiovascular $(\mathrm{RCV})^{3}$.

La obesidad abdominal ha estado en el centro de lo que ha sido llamado Síndrome Metabólico, entidad que implica un alto RCV. La complejidad fisiopatológica y semántica que rodea al Síndrome Metabólico, concepto que aún continua debatiéndose después de su introducción hace ya más de 30 años, quizás está reflejada por el uso de Arnaiz y cols., del término "riesgo cardiometabólico", en el título de su trabajo, y en porciones de éste ${ }^{4}$.

El uso intercambiable que hacen los autores de este término con el de Síndrome Metabólico refleja las incertidumbres conceptuales que aun persisten con respecto a este tema. Las posturas contrastantes del American Diabetes Association, recomendando el abandono de este término, y la de la American Heart Association y del National Heart, Lung and Blood Institute, endorsándolo, son sintomáticas de este debate todavía inconcluso ${ }^{5,6}$.
En definitiva, aún no existen evidencias para decidir si el Síndrome Metabólico es realmente una entidad fisiopatológica que obedece a una cadena causal única y específica o simplemente es una forma de reunir factores de RCV reconocidos bajo un mismo techo, pero con mecanismos causales independientes. Desde el estudio de Framingham se sabe que el RCV en los individuos que presentan múltiples factores de riesgo es progresivamente mayor en la medida que estos se van agregando: en un individuo de 50-60 años portador de un conglomerado de factores que incluya colesterol LDL elevado, tabaquismo, hipertensión y diabetes mellitus, la posibilidad de un evento coronario en un plazo de 10 años es 400 a $600 \%$ veces mayor que en la ausencia de ellos ${ }^{7}$. Sin embargo, la reducción del riesgo en ese individuo no es mayor de un 20 a 30 $\%$ controlando esos mismos factores de riesgo en forma aislada ${ }^{8,9}$. Esta discrepancia sugiere que hay factores aún no identificados que contribuyen a un efecto posiblemente sinergístico de los factores de RCV. La capacidad predictora que tiene el Síndrome Metabólico podría simplemente reflejar ese fenómeno potenciador de una constelación de factores que iría más allá del efecto sumatorio de ellos. Este efecto sugiere cadenas causales comunes en la que factores inflamatorios han sido identificados junto a otros aun por definir. Resistencia a la Insulina y acumulación de tejido adiposo visceral, ambos mediados, al menos parcialmente, por una expresión aumentada de adipo citokinas (adiponectina, y más recientemente visfatina) han sido propuestos como elementos claves en la patogenia de este conglomerado de factores que incluye a la diabetes, obesidad abdominal, trastornos lipídicos (colesterol HDL bajo, hipertrigliceridemia) e hipertensión arterial ${ }^{10}$.

Cual es el mejor método antropométrico para medir el impacto de la obesidad en el RCV no está aun de- 
finitivamente establecido, pero el peso de la evidencia actual favorece aquellos que miden su distribución abdominal. Sin embargo, factores étnicos pueden influir en la mayor o menor sensibilidad y/o especificidad de la capacidad predictiva de los diferentes métodos. En Chile la razón cintura/estatura (RCE) ha sido previamente utilizada en adultos con una predicción del RCV superior a otros parámetros antropométricos ${ }^{11}$. La RCE ofrece teóricamente la ventaja de que al incorporar la estatura, capturaría otra dimensión del riesgo posiblemente relacionado a condiciones adversas en las etapas tempranas de la vida ${ }^{12,13}$.

Los autores usan una definición de Síndrome Metabólico tomada de Cook, y cols., a su vez basada en el criterio propuesto por el ATP III para adultos en USA ${ }^{14}$.

Cook y cols., utilizaron esta definición en la encuesta NAHNES 1988-1994 que incluyó a un total de 2.430 adolescentes con edades entre 12 y 19 años, (aunque la mayoría entre 15 y 19); la representación étnica incluyó alrededor de un $30 \%$ de blancos, negros y México-Americanos, respectivamente.

El trabajo de Arnaiz y cols., incluyó escolares con una edad promedio de 10.8 años, probablemente la mayoría en la pre-adolescencia, inferior a la del estudio de Cook y cols. por lo que cabe preguntarse, ¿en qué medida estas definiciones basadas en valores tomados de una población de adultos norteamericanos son aplicables a poblaciones adolescentes de ese país por una parte, y por otra a escolares chilenos con una edad inferior y composición

\section{Referencias:}

1. Gould SJ. The Mismeasure of Man (New York: W. W. Norton, 1981

2. Sheldon WH. The varieties of human physique: An introduction to constitutional psychology (New York: Harper \& Brothers, 1940).

3. Puska P, Nishida C, Porter D. World Health Organization 2008 Report. Global Strategy on Diet, Physical Activity and Health. Obesity and Overweight. Disponible en: http://www.who.int/dietphysicalactivity/media/en/gsfs_obesity.pdf.

4. Arnaiz PG,Acevedo M, Díaz C, Bancalari R, Barja S,Aglony M, et al. Razón cintura estatura como predictor de riesgo cardiometabólico en niños. Rev Chil Cardiol 2010; 29: 281 - 288.

5. Kahn R, Buse J, Ferrannini E, Stern M. The metabolic syndrome: time for a critical appraisal. Joint statement from the American Diabetes Association and the European Association for the Study of Diabetes. Diabetologia. 2005; 48: 1679-83.

6. Grundy SM, Cleeman JI, Daniels SR, Donato KA, Eckel RH, étnica diferente a la de éstos?. Sin embargo, cabe hacer notar, que a pesar de las diferencias en edades y etnias, las prevalencias de 3 o $>$ factores de riesgo (o de Síndrome Metabólico de acuerdo a la definición utilizada) fueron muy similares (28.7\% en el estudio de Cook et al, $29.9 \%$ en el de Arnaiz y cols. para los percentiles de peso >95). Esto apoyaría indirectamente a la existencia de una cadena causal del riesgo cardiovascular ligada a la acumulación abdominal de tejido adiposo quizás independiente de factores etarios o étnicos.

En resumen, los autores lograron identificar en niños chilenos, utilizando una metodología de muestreo y análisis estadístico apropiadas, a la RCE como un predictor de buena sensibilidad y especificidad (aunque no superior al IMC en este estudio) de factores de riesgo cardiovascular agrupados en el conglomerado del Síndrome Metabólico. Tal como lo comentan los autores, este método ha sido validado previamente en nuestro medio como un parámetro antropométrico de valor en la predicción del riesgo cardiovascular en adultos

El mayor mérito del trabajo de Arnaiz y cols., no obstante las limitaciones inherentes al estado actual del tema y en la ausencia de definiciones mejores del Síndrome Metabólico y en particular en el cambiante escenario de la niñez y adolescencia, está en alertar a los profesionales de la salud encargados de la atención primaria de la existencia de un marcador de riesgo cardiovascular en una edad temprana que puede utilizarse en cualquier lugar del país sin mayores instrumentos que una cinta de medir.
Franklin BA, et al. Diagnosis and management of the metabolic syndrome: an American Heart Association/National Heart, Lung, and Blood Institute Scientific Statement. Circulation. 2005; 112: 2735-52.

7. Wilson PW, D'Agostino RB, Levy D, Belanger AM, Silbershatz H, Kannel WB.Prediction of Coronary Heart Disease Using Risk Factor Categories. Circulation. 1998; 97: 1837-1847.

8. Jamerson K, Bakris GL, Dahlöf B, Pitt B, Velazquez E, Gupte $\mathrm{J}$, et al. Exceptional early blood pressure control rates: the ACCOMPLISH trial. Blood Press 2007; 16: 80-6.

9. Shepherd J, Cobbe SM, Ford I, Isles CG, Lorimer AR, MacFarlane PW, et al. Prevention of coronary heart disease with pravastatin in men with hypercholesterolemia. West of Scotland Coronary Prevention Study Group. N Engl J Med 1995; 333:1301-1310.

10. Moschen AR, Kaser A, Enrich B, Mosheimer B, Theurl M, Niederegger H, Visfatin, an adipocytokine with proinflammatory and immunomodulating properties. J Immunol. 2007; 178:1748-58

11. Koch E, Otárola A, Romero T, Manríquez L, Paredes M, KIrs- 
La cinta de medir como t cnica predictiva: la raz n Cintura-Estatura, el s ndrome metab lico y el riesgo cardiovascular.

Romero $T$, et al.

chbaum A, et al. Mediciones antropométricas y riesgo de sufrir un evento cardiovascular en población chilena. Resultados del proyecto San Francisco. Rev Chil Cardiol 2006; 25: 171-182.

12. Hebert PR, Rich-Edwards JW, Manson JE, Ridker PM, Cook NR, O'Connor GT, et al. Height and incidence of cardiovascular disease in male physicians. Circulation. 1993; 88: 1437-1443.

13. Koch E, Romero T, Romero C, Manríquez L, Paredes M, Ahu- mada C, Aguilera H. Impact of Height on Cardiovascular Risk Factors and All-Cause Mortality: A Prospective Study in a Cohort of Chilean Adults. Circulation 2010; 122: e2.

14. Cook S, Weitzman M, Auinger P, Nguyen M, Dietz WH. Prevalence of a metabolic syndrome phenotype in adolescents: findings from the third National Health and Nutrition Examination Survey, 1988-1994. Arch Pediatr Adolesc Med 2003; 157: 821-7. 